\title{
Research of distribution route optimization based on adaptive ant colony algorithm cloud logistics
}

\author{
Zhigao Chen \\ (Hu Nan vocational institute of science \& technology, Changsha, China, 410018) \\ Chenzhigao7621414@163.com
}

Keywords: Path Optimization; Adaptive ant colony algorithm; Cloud logistics;

\begin{abstract}
Cloud Manufacturing is developed in the cloud computing, which is based on the idea of "Made in service" recently. Cloud manufacturing mode connect all sorts of manufacturing resources and capability together and form standard manufacturing services which customers can obtain according to their demand at any time. Logistics mode should coincide with manufacturing mode for the certain correlation between logistics and manufacturing process, so the production and development of cloud manufacturing mode had the logistics mode reformed. In this paper we present a cloud manufacturing service composition method considering execution reliability based on ant algorithm, to optimize the use of composite service execution path to save the costs.
\end{abstract}

\section{Introduction}

Under the global economy, competition and cooperation among the enterprises become a normal state, but enterprises hope to make full use of resources to reduce the production cost and make customers satisfy. For this purpose, along with the new IT technology development, such as high performance computing arid Internet of things etc, a new manufacturing mode-- cloud manufacturing was formed[1].

Cloud manufacturing comprehensively uses various information technology, manufacturing technology and management technology, through virtualization of manufacturing hardware and software resources which are massive, heterogeneous, distributed and the manufacturing whole life circle oriented, to form a unified manufacturing cloud service pool, through building a cloud manufacturing service platform to uniformly, centrally, on-demand and intelligently manage and operate manufacturing cloud services, thereby to provide reliable, high quality, cheap and on-demand manufacturing services for various users, sequentially to support convenient, dynamic business collaboration between enterprises, at last to promote manufacturing resources optimization allocation within the scope of the wide area[2].

Cloud manufacturing needs to run in high-speed network to play its advantages, the research and application of efficient routing mechanism can improve the efficiency of scheduling speed resources.

\section{Characteristic of cloud manufacturing}

\section{A. Cloud logistics}

The On the cloud platform, all of the logistics company, agent service providers, equipment manufacturers, industry associations, regulatory agencies, industry, media, legal structure and so focused on cloud integration into resource pools, various resources and interact with each other to show, on-demand communication, reach intention to reduce costs and improve efficiency.

$B$. Features of ant algorithm

The ant algorithm is proposed by the Italian scholar Marco Dorigo in 1992, a parallel and efficient evolutionary algorithm, the core idea is: ants will left a " pheromone" chemical substances in the path searching for food, these" pheromone" can provide heuristic information where selected on walking routes, for the follow-up ants to find food, as the constant updating of pheromone, optimal path can be found from the nest to the food in a relatively short period of time. The 
algorithm has the advantages of high parallel, convergence speed, and has Gained some satisfactory experimental results, in the traveling salesman problem, routing and scheduling problems, but the standard ant algorithm is easy to fall into local optimal solution[4].

$C$.Application in cloud manufacturing of ant algorithm

Cloud manufacturing is a service-oriented, knowledge-based smart manufacturing system with high efficiency and low energy consumption. In a cloud manufacturing system, state-of-the-art technologies such as informatized manufacturing technology, cloud computing, Internet of Things, semantic Web, high-performance computing, and cloud manufacturing are integrated. By extending and shifting existing manufacturing and service systems, manufacturing resources and capabilities are virtualized and oriented towards service provision. In cloud manufacturing, pervasive and efficient sharing and coordination of resources and capabilities can be achieved by their unified and centralized intelligent management and operation. Cloud manufacturing provides the whole manufacturing lifecycle with secure, reliable, high quality, and on-demand services at low prices through networked systems[5].

\section{Adaptive ant algorithm Operating Model in Cloud Manufacturing}

The ant algorithm can be applied to cloud manufacturing methods are as follows:

Firstly, each node in the cloud environment was treated as the point in the map abstractly, a certain node was set as the start point, will was searched by the ant as the "food" finally, namely to complete the routing process.

Secondly, the shortest path can be found by the ant Algorithm on the origin to the destination.

Third, adjust the search methods, optimization algorithms for optimal path.

A. Procedure of Ant Algorithm

Firstly, each node in the cloud environment is abstracted as a connected graph point to determine a node as a starting point, the final node of the visit as "food" by the ants to search. Routing process was completed when the ants had found the target in the traditional ant algorithm, Filmon was reserved in line which the ants searched, the algorithm has been modified in this paper that the film on was reserved on each node traversed by the ants. The $\tau$ i represents the amount of information, $\mathrm{H}$ represents the network capacity values the node can carry, Threshold is set to di, if di $>\mathrm{H}$, hosted by the node network traffic has exceeded the network capacity, this means that the congestion would be coming on the node ,then the new business transmission can not be longer allowed in the path. The choice of the new path must be started in order to bypass the sections in upcoming congestion, a new sub-optimal path would be Created.

With the establishment and the end of the network session, the amount of information on each node would change when each cycle completed, the Fireman on each node Adjusted as(1), (2) :Among them, the k-th ant in the cycle to stay in the pixels on the filmon.

$$
\tau_{i}(t+1)=\rho \cdot \tau_{i}(t)+\Delta \tau_{i} \text { (1) } \quad \Delta \tau_{i}=\sum_{i=1}^{n+1} \Delta \tau_{i}^{k}(2)
$$

\section{B. Experiment}

Figure 1 is a simulation example of a network of choice in this article, a total of 24nodes in the graph, it is assumed from the source node 1 to node 23, and the source node to node 24, two paths through the data routing experiment.

Step1: designed to be adaptive ant algorithm to find the shortest path to node $1 \rightarrow 23$; here to take $\alpha=1, \beta=2, \rho=0.8, Q=1000$, the initial value $\tau \mathrm{min}=60$. Get $1 \rightarrow 23$ of the shortest path is (1): $1 \rightarrow 3 \rightarrow 6 \rightarrow 11 \rightarrow 16 \rightarrow 20 \rightarrow 23$, 15 flow (minimum segment capacity) 4 ;

Step2: the capacity of each segment of the shortest path 1 minus the smallest segment of the shortest path capacity, the results of $16 \rightarrow 20$ segment of the capacity is 0 , the segment identified as the bottleneck segment, t he segment most prone to congestion ;

Step3: According to the capacity change and the connection matrix changes to look for the source node and destination node 23 the shortest path available to the new 
shortest path. (2) $: 1 \rightarrow 4 \rightarrow 7 \rightarrow 12 \rightarrow 17 \rightarrow 19 \rightarrow 20 \rightarrow 23$, 18 flow rate, and similarly the capacity of each segment of the shortest path (2) subtract the smallest segment of the shortest path capacity, the result of the capacity of the $4 \rightarrow 7$ segment 0 , that segment is identified as unavailable, repeat the same steps to find the shortest path

(3)change Network: $1 \rightarrow 4 \rightarrow 9 \rightarrow 14 \rightarrow 19 \rightarrow 20 \rightarrow 23$, length 19 , flow rate 3 ;

(4): $1 \rightarrow 2 \rightarrow 5 \rightarrow 10 \rightarrow 15 \rightarrow 18 \rightarrow 20 \rightarrow 23$, length 21 , flow rate of 5 ;

SteP4: could not find a feasible route of the destination node of a source node $\rightarrow$ (or less than the number of shortest path to the default limit), the source node or destination node will become an isolated point, out of the end of the loop;

Step5: will find the shortest path sequence as the source node 1 $\rightarrow$ purpose node 23optional routing tables, the same method can be found in the $9 \rightarrow 15$ optional routing tables. As the experiment shown, the adaptive ant algorithm constructed in this paper had got the data listed in table 1.

As the experimental results, dynamic optimal routings from node $1 \rightarrow 23$ and $9 \rightarrow 24$, There are four paths of Optimal routing from node $1 \rightarrow 23$,and four paths of Optimal routing from $9 \rightarrow 24$ as the same, According to the provisions of the preferential routing priority, In order to achieve a dynamic optimal routing because of the network congestion or partial failure.

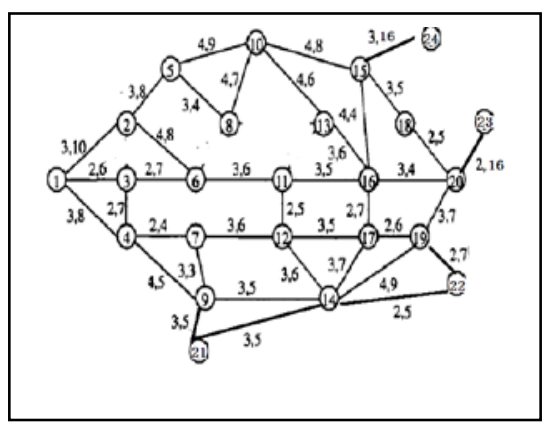

Figure 1 node topology
Table 1 dynamic optimal routing

\begin{tabular}{|cclrr|}
\hline \multicolumn{2}{|c|}{ Source $\rightarrow$ target order selected Path } & shortest path & flow \\
\hline $1 \rightarrow 23$ & 1 & $1 \rightarrow 3 \rightarrow 6 \rightarrow 11 \rightarrow 16 \rightarrow 20 \rightarrow 23$ & 15 & 4 \\
& 2 & $1 \rightarrow 4 \rightarrow 7 \rightarrow 12 \rightarrow 17 \rightarrow 19 \rightarrow 20 \rightarrow 23$ & 18 & 4 \\
& 3 & $1 \rightarrow 4 \rightarrow 9 \rightarrow 14 \rightarrow 19 \rightarrow 20 \rightarrow 23$ & 19 & 3 \\
& 4 & $1 \rightarrow 2 \rightarrow 5 \rightarrow 10 \rightarrow 18 \rightarrow 20 \rightarrow 23$ & 21 & 5 \\
& & & \\
& & & \\
\hline $9 \rightarrow 24$ & 1 & $9 \rightarrow 14 \rightarrow 17 \rightarrow 16 \rightarrow 15 \rightarrow 24$ & 15 & 4 \\
& 2 & $9 \rightarrow 14 \rightarrow 19 \rightarrow 20 \rightarrow 18 \rightarrow 15 \rightarrow 24$ & 18 & 1 \\
& 3 & $9 \rightarrow 7 \rightarrow 6 \rightarrow 8 \rightarrow 10 \rightarrow 15 \rightarrow 24$ & 19 & 3 \\
4 & $9 \rightarrow 4 \rightarrow 3 \rightarrow 2 \rightarrow 5 \rightarrow 10 \rightarrow 15 \rightarrow 24$ & 21 & 5 \\
\hline \multicolumn{5}{c}{} \\
\end{tabular}

\section{Conclusion}

Cloud logistics is a new logistics mode which can make a variety of logistics resources and capabilities virtualization integrated and intelligent management and operation, and service for different customers in order to achieve efficient coordination and win-win. This paper has a study based on the analysis on cloud manufacturing resource scheduling and discovery process, builds a practical and useful manufacturing scheduler model and scheduling policy on ant algorithm. We got the following conclusions:

(1) Comparison and principle of route optimization: basic principles block diagrams and operating framework of intelligent algorithm are introduced and compared, which makes the preparation for the algorithm proposed later.

(2) Basic ant colony algorithm: principles, flow diagram of solving TSP problems and shortage for solving practical problems of basic algorithm are introduced improved algorithm-adaptive ant colony algorithm is proposed.

(3) Research on route optimization: algorithm is taken to design route optimization system.

\section{References}

[1] S. D. Shtovba. Ant Algorithms: Theory and Applications[J]. Programming and Computer Software . 2005 (1) 
[2] Daniel Merkle,Martin Middendorf. Ant Colony Optimization with Global Pheromone Evaluation for Scheduling a Single Machine[J]. Applied Intelligence . 2003 (1)

[3] A Brief Survey on the Security Model of Cloud Computing[A]. Proceedings of the Ninth International Symposium on Distributed Computing and Applications to Business,Engineering and Science[C]. 2010.

[4] Research and Implementation of Future Network Computer based on Cloud Computing[A]. Proceedings of 2010 Third International Symposium on Knowledge Acquisition and Modeling (KAM 2010)[C]. 2010.

[5] Nezamabadi-pour H,Saryazdi S,and Rashedi E.Edge detection using ant algorithm[J].Soft Comput,2006,10(7): 623-628. 